\title{
Efficacy and Safety of Budesonide/Glycopyrrolate/ Formoterol Fumarate Metered Dose Inhaler Formulated Using Co-Suspension Delivery Technology in Japanese Patients with COPD: A Subgroup Analysis of the KRONOS Study
}

This article was published in the following Dove Press journal: International Journal of Chronic Obstructive Pulmonary Disease

\author{
Masakazu Ichinose, \\ Yasushi Fukushima, (D) ${ }^{2}$ \\ Yoshikazu Inoue, $\mathbb{D i D}^{3}$ \\ Osamu Hataji, ${ }^{4}$ \\ Gary T Ferguson, ${ }^{5}$ Klaus F Rabe, ${ }^{6}$ \\ Nobuya Hayashi, $\mathbb{1 D}^{7}$ \\ Hiroshi Okada, $\mathbb{1}^{7}$ \\ Mami Takikawa, ${ }^{7}$ Eric Bourne, ${ }^{8}$ \\ Shaila Ballal, \\ Kiernan DeAngelis, (iD) ${ }^{10}$ \\ Magnus Aurivillius, "I \\ Paul Dorinsky, (1D) \\ Colin Reisner (1D) \\ 'Department of Respiratory Medicine, Tohoku \\ University Graduate School of Medicine, Sendai, \\ Japan; ' ${ }^{2}$ Department of Internal Medicine, Fukuwa \\ Clinic, Tokyo, Japan; ${ }^{3}$ Clinical Research Center, \\ National Hospital Organization, Kinki-Chuo Chest \\ Medical Center, Osaka, Japan; ${ }^{4}$ Respiratory Center, \\ Matsusaka Municipal Hospital, Matsusaka, Japan; \\ ${ }^{5}$ Pulmonary Research Institute of Southeast \\ Michigan, Farmington Hills, MI, USA; '́LungenClinic \\ Grosshansdorf and Christian-Albrechts University \\ Kiel, Airway Research Center North, Member of \\ the German Center for Lung Research (DZL), \\ Grosshansdorf, Germany; ${ }^{7}$ AstraZeneca K.K. \\ Osaka, Japan; ${ }^{8}$ AstraZeneca, Durham, NC, USA; \\ ${ }^{9}$ AstraZeneca, Morristown, NJ, USA; ${ }^{10}$ Formerly of \\ AstraZeneca, Durham, NC, USA; " AstraZeneca, \\ Gothenburg, Sweden
}

Correspondence: Masakazu Ichinose Department of Respiratory Medicine, Tohoku University Graduate School of

Medicine, Sendai, Japan

Tel +8I-22-7I7-8534

Email ichinose@rm.med.tohoku.ac.jp
Background: KRONOS, a Phase III, multicenter, randomized, double-blind study (NCT02497001) conducted in Canada, China, Japan, and the USA, assessed the efficacy and safety of budesonide/glycopyrrolate/formoterol fumarate metered dose inhaler (BGF MDI), a triple fixed-dose combination therapy, relative to dual therapies in patients with moderate-to-very severe COPD. Here we present findings from the Japanese subgroup of KRONOS.

Methods: Patients received BGF MDI 320/18/9.6 $\mu$ g, glycopyrrolate/formoterol fumarate

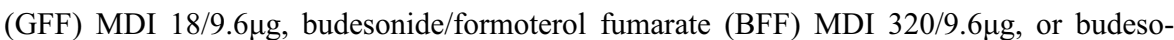
nide/formoterol fumarate dry powder inhaler (BUD/FORM DPI) 400/12 $\mu$ g twice-daily for 24 weeks. The primary endpoint was the change from baseline in morning pre-dose trough forced expiratory volume in $1 \mathrm{~s}\left(\mathrm{FEV}_{1}\right)$ over Weeks 12-24. Symptoms, quality of life, exacerbations, and safety were also assessed.

Results: In total, 416 Japanese patients (21.9\% of the global KRONOS population) were randomized and treated with BGF MDI ( $n=139)$, GFF MDI ( $\mathrm{n}=138)$, BFF MDI ( $\mathrm{n}=70)$, or BUD/FORM DPI ( $n=69)$. Nominally significant improvements in the change from baseline in morning pre-dose trough $\mathrm{FEV}_{1}$ over Weeks 12-24 were observed for BGF MDI vs GFF MDI (least squares mean [LSM] difference $37 \mathrm{~mL}, 95 \%$ confidence interval [CI] 3, 72; $P=0.0337)$ and BFF MDI $(67 \mathrm{~mL}$; $95 \%$ CI 25, 109; $P=0.0020$ ). Treatment with BGF MDI led to a nominally significant reduction in the rate of moderate/severe exacerbations vs GFF MDI (rate ratio $0.40,95 \%$ CI $0.19,0.83 ; P=0.0142$ ). Compared with dual therapies, numerical improvements were observed with BGF MDI for Transition Dyspnea Index focal score and the change from baseline in Evaluating Respiratory Symptoms in COPD total score $(P \leq 0.3899)$. All treatments were generally well tolerated.

Conclusion: BGF MDI nominally significantly improved lung function and numerically improved symptoms vs GFF MDI and BFF MDI. BGF MDI nominally significantly reduced exacerbations vs GFF MDI in Japanese patients with COPD. Efficacy and safety findings were generally comparable to those in the global KRONOS population.

Keywords: co-suspension delivery technology, ICS/LAMA/LABA, inhaled corticosteroid, long-acting muscarinic antagonist, long-acting $\beta_{2}$-agonist, Japan

\section{Introduction}

The Nippon COPD Epidemiology study, conducted in 2000, estimated that at least $8.6 \%$ of Japanese adults (aged $\geq 40$ years) have airflow limitation indicative of 
COPD. ${ }^{1}$ In 2015, COPD was the tenth leading cause of death in Japan; ${ }^{2}$ however, public awareness of the disease is low, with fewer than $20 \%$ of adults reporting that they were aware of COPD in a 2012 survey. ${ }^{3}$ Japan has the highest percentage of adults over the age of 65 in the world, ${ }^{4}$ and thus the burden of COPD is expected to grow with the aging of the population.

Treatment of COPD is typically initiated with a single bronchodilator (long-acting muscarinic antagonist [LAMA] or long-acting $\beta_{2}$-agonist [LABA]), with the addition of a second bronchodilator and/or an inhaled corticosteroid (ICS), if necessary, to control symptoms and decrease the risk of exacerbations. ${ }^{5}$ Triple ICS/LAMA/LABA combinations are currently recommended by the Global Initiative for Chronic Obstructive Lung Disease (GOLD) as a step-up treatment for patients who experience further exacerbations on dual therapy (with step-up from LAMA/LABA recommended particularly for those with blood eosinophil levels $\geq 100$ cells $/ \mathrm{mm}^{3}$ ), or have persistent breathlessness on ICS/ LABA therapy. ${ }^{5}$ Notably, the Japanese Respiratory Society (JRS) only recommends ICS-containing therapies for patients with COPD who have features of asthma-COPD overlap (ACO), ${ }^{6}$ which include bronchodilator responsiveness $>400 \mathrm{~mL}$ and elevated blood eosinophil levels $\left(\geq 300\right.$ cells $\left./ \mathrm{mm}^{3}\right){ }^{7,8}$ In line with these recommendations, ICS use tends to be lower in Japanese patients with COPD compared to global patient populations, based on observations from international clinical trials. ${ }^{9,10}$

A greater benefit of triple therapies vs. dual therapies on lung function, symptoms, and exacerbations has been supported by several recent studies; ${ }^{11-15}$ however, triple therapy has not been extensively studied in Japanese patients with COPD, who may differ from Western patients in their demographics, clinical characteristics, and pharmacological treatment history. ${ }^{16-18}$ Budesonide/ glycopyrrolate/formoterol fumarate metered dose inhaler (BGF MDI) is a triple fixed-dose combination of an ICS, a LAMA, and a LABA formulated using co-suspension delivery technology that is currently in global development for COPD. In a pivotal 24-week, Phase III, multicenter trial (KRONOS; NCT02497001), which included patients from Japan, BGF MDI showed benefits on lung function, symptoms, and exacerbations vs. corresponding LAMA/LABA and ICS/LABA therapies, with a similar safety profile. ${ }^{15}$ Here we report findings from the Japanese subgroup of KRONOS to assess the effects of BGF MDI in Japanese patients with COPD and discuss these in the context of results from the global population. ${ }^{15}$

\section{Materials and Methods}

\section{Study Design}

Details of the study design have been previously published. ${ }^{15}$

Briefly, KRONOS was a double-blind, parallel-group, multicenter, Phase III randomized trial, conducted in Canada, China, Japan, and the USA. Patients were randomized 2:2:1:1 to receive BGF MDI 320/18/9.6 $\mu$ g, glycopyrrolate/ formoterol fumarate (GFF) MDI 18/9.6 $\mu \mathrm{g}$, budesonide/formoterol fumarate (BFF) MDI 320/9.6 $\mu \mathrm{g}$, or open-label budesonide/formoterol dry powder inhaler (BUD/FORM DPI; Symbicort Turbuhaler ${ }^{\circledR}$ ) 400/12 $\mu \mathrm{g}$ twice-daily for 24 weeks. For all treatments, each dose was administered as two inhalations. BUD/FORM DPI 400/12 $\mu \mathrm{g}$ was administered as two inhalations of 200/6 $\mu \mathrm{g}$ (metered dose, corresponding to a total daily delivered dose of $320 / 9 \mu \mathrm{g}$ ). The $18 \mu \mathrm{g}$ dose of glycopyrrolate and $9.6 \mu \mathrm{g}$ dose of formoterol fumarate are equivalent to $14.4 \mu \mathrm{g}$ and $10 \mu \mathrm{g}$, respectively, of glycopyrronium and formoterol fumarate dihydrate (delivered doses).

The study was conducted in accordance with Good Clinical Practice, including the Declaration of Helsinki. The protocol and informed consent form were approved by appropriate institutional review boards (full details have been published). ${ }^{15}$ All patients provided written informed consent before screening.

\section{Patients}

Inclusion and exclusion criteria for KRONOS have been published. ${ }^{15}$ Briefly, patients were current or former smokers, 40-80 years of age, with an established clinical history of COPD, which for Japanese patients was defined according to the JRS guidelines ${ }^{19}$ and confirmed by the investigator. Patients had moderate-to-very severe COPD, defined as a postbronchodilator forced expiratory volume in $1 \mathrm{~s}\left(\mathrm{FEV}_{1}\right) \geq 25 \%$ and $<80 \%$, according to predicted normal values using reference norms for Japan. ${ }^{19,20}$ All patients were symptomatic (COPD Assessment Test [CAT] score $\geq 10$ ) despite receiving two or more inhaled maintenance therapies for at least 6 weeks before screening. There was no requirement for a history of COPD exacerbations in the previous year. Exclusion criteria included, but were not limited to, a current diagnosis of asthma or any other respiratory disorder (other than COPD) which, in the opinion of the investigator, could influence the study results.

\section{Study Endpoints}

This manuscript reports findings according to the Japan/ China regulatory approach, in which most endpoints were assessed over Weeks 12-24. Data for the global KRONOS 
study population are presented for comparison. Lung function endpoints were change from baseline in morning predose trough $\mathrm{FEV}_{1}$ over Weeks 12-24 (primary) and over 24 weeks (secondary). Post-dose spirometry measurements were not conducted in Japan, and therefore the additional secondary lung function endpoints from the Japan/China approach $\left(\mathrm{FEV}_{1}\right.$ area under the curve from 0 to $4 \mathrm{hrs}$ [AUC ${ }_{0-4}$ ] over Weeks 12-24 and peak change from baseline in $\mathrm{FEV}_{1}$ within 4 hrs post-dosing over Weeks 12-24) are not available in the Japanese population.

Secondary symptom and quality of life endpoints included Transition Dyspnea Index (TDI) focal score over Weeks 12-24, change from baseline in St George's Respiratory Questionnaire (SGRQ) total score over Weeks 12-24, change from baseline in mean daily number of puffs of rescue medication over 24 weeks, and time to clinically important deterioration (CID; defined as one of the following: $a \geq 100 \mathrm{~mL}$ decrease from baseline in trough $\mathrm{FEV}_{1} \mathrm{OR} \mathrm{a} \geq 4$ point increase from baseline in SGRQ total score OR a TDI focal score of -1 point or less OR a treatment-emergent moderate or severe COPD exacerbation occurring up to Week 24). Other efficacy endpoints included the rate of moderate or severe COPD exacerbations over 24 weeks and change from baseline in mean daily Evaluating Respiratory Symptoms (E-RS) total score over Weeks 12-24. CID and moderate/severe exacerbations were defined in KRONOS, as reported by Ferguson et al. ${ }^{15}$

Safety was assessed via adverse event monitoring, 12-lead electrocardiograms, clinical laboratory testing, and vital sign measurements. Cases of pneumonia and major adverse cardiovascular events (MACE) were adjudicated by an external clinical endpoint committee (CEC) to ensure that appropriate pre-defined and clinically consistent criteria were met for these outcomes. Safety data were reviewed by an external data monitoring committee.

\section{Statistical Analysis}

Although the Japanese subgroup analysis was pre-specified, no adjustments for multiplicity were made. Therefore, all $P$-values and statistical significance reported in this manuscript are nominal. The Japanese modified intent-to-treat (mITT) and safety populations were subsets of the KRONOS mITT and safety populations that included the patients enrolled at sites in Japan. The comparisons of interest for the Japan/China statistical approach were BGF MDI vs. BFF MDI and vs. GFF MDI (both for superiority) and BFF MDI vs. BUD/FORM DPI (for non-inferiority; assessed in the global population only).

The primary endpoint was analyzed using a linear repeated measures model which included the following covariates: baseline trough $\mathrm{FEV}_{1}, \%$ reversibility to albuterol, baseline eosinophil count, visit, treatment, treatment-by-visit interaction, and ICS use at screening. Analyses of secondary endpoints used similar models with their respective baseline measures as covariates. Time to CID was analyzed using a Cox proportional hazards model, and the rate of moderate or severe exacerbations was analyzed using a negative binomial model. A post hoc analysis of the rate of moderate or severe exacerbations according to baseline reversibility to albuterol was also performed in the Japanese population. Further details of the statistical analysis have been previously published. ${ }^{15}$

\section{Results \\ Study Population}

Of the 1902 patients who were randomized in the KRONOS study globally, a total of 416 (21.9\%) were randomized and treated in Japan (Figure 1). All were included in the Japanese safety and mITT populations (mean age 69.5 years, $94.2 \%$ male, mean post-albuterol FEV $_{1} 51.9 \%$ of the predicted normal value; Table 1 ).

The most common COPD medication in the Japanese population at study entry was a LAMA plus a LABA (66.6\%) followed by triple therapy consisting of an ICS, a LAMA, and a LABA (19.7\%); in total, $31.5 \%$ of patients were receiving an ICS-containing therapy at screening. Approximately half of Japanese patients had a blood eosinophil count $\geq 150$ cells $/ \mathrm{mm}^{3}$ (52.4\%) and a minority had counts $\geq 300$ cells $/ \mathrm{mm}^{3}$ (13.2\%). Reversibility (defined as improvement in $\mathrm{FEV}_{1}$ of $\geq 12 \%$ and $\geq 200 \mathrm{~mL}$ following administration of albuterol) was observed in $37.5 \%$ of Japanese patients, and $3.6 \%$ of patients had reversibility $\geq 400 \mathrm{~mL}$. Overall, most Japanese patients had no history of COPD exacerbations in the year prior to study entry $(80.0 \%)$. The proportion of patients in the Japanese population who had experienced $\geq 1$ exacerbation in the past 12 months was highest in the BGF MDI group (25.9\%) and comparable across the dual therapy groups (range: $15.9-17.4 \%$; Table 1).

\section{Lung Function}

For the primary endpoint of change from baseline in morning pre-dose trough $\mathrm{FEV}_{1}$ over Weeks 12-24, nominally significant improvements for BGF MDI vs. GFF MDI (least squares mean [LSM] difference $37 \mathrm{~mL}, 95 \%$ confidence interval $[\mathrm{CI}] 3,72 \mathrm{~mL} ; P=0.0337)$ and vs. BFF MDI (LSM difference $67 \mathrm{~mL}, 95 \%$ CI 25, $109 \mathrm{~mL}$; $P=0.0020$; Table 2, Figure 2) were observed in Japanese patients. Similarly, in the global population, BGF MDI 


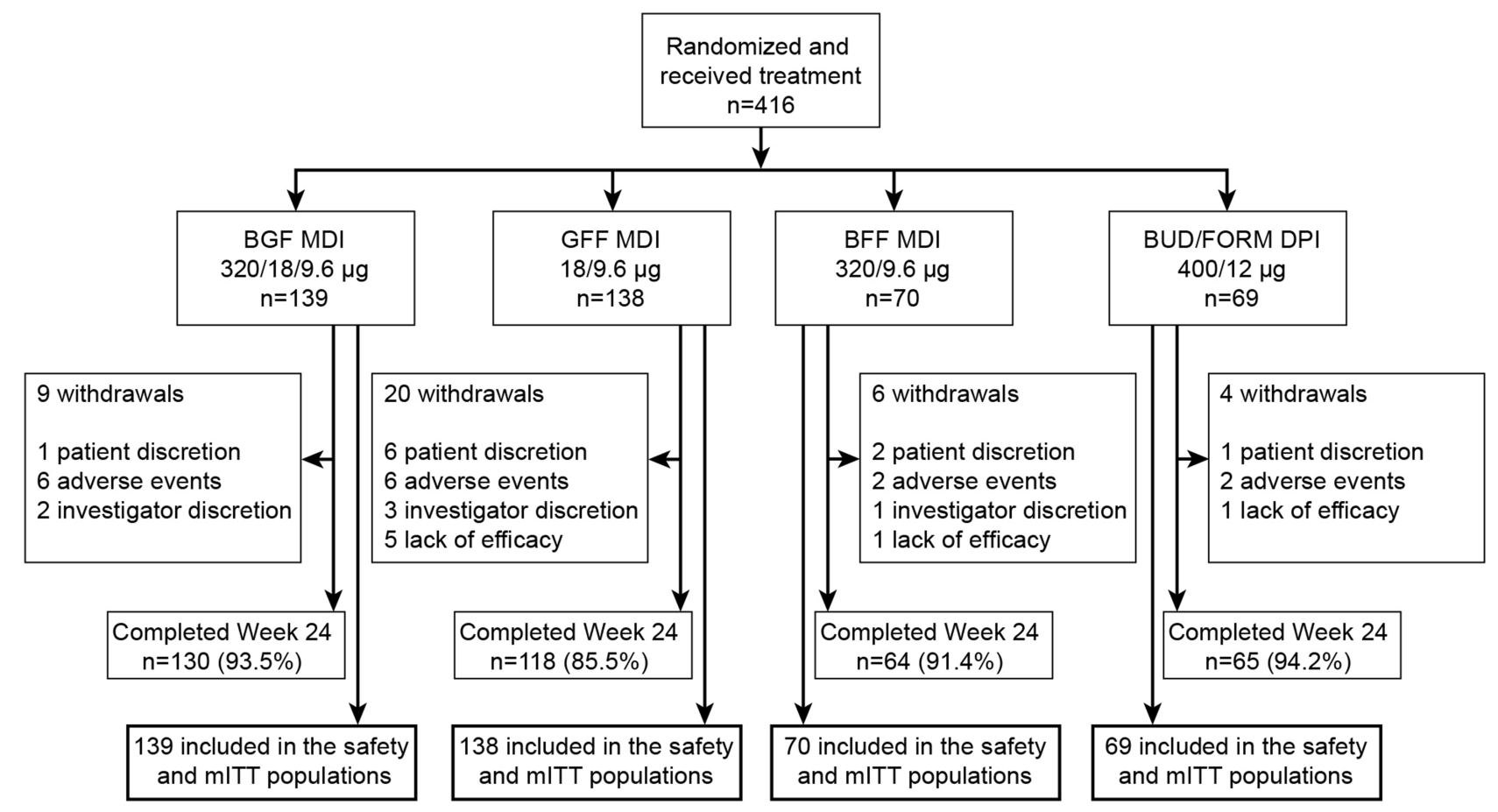

Figure I Patient disposition.

Abbreviations: BFF, budesonide/formoterol fumarate; BGF, budesonide/glycopyrrolate/formoterol fumarate; BUD/FORM DPI, budesonide/formoterol fumarate dry powder inhaler; GFF, glycopyrrolate/formoterol fumarate; MDI, metered dose inhaler; mITT, modified intent-to-treat.

significantly improved morning pre-dose trough $\mathrm{FEV}_{1}$ over Weeks 12-24 compared to GFF MDI (LSM difference $20 \mathrm{~mL}, 95 \% \mathrm{CI} 1,39 \mathrm{~mL} ; P=0.0424)$ and BFF MDI (LSM difference $77 \mathrm{~mL}, 95 \%$ CI 53, $100 \mathrm{~mL} ; P<0.0001$; Table 2). BFF MDI was non-inferior to BUD/FORM DPI for the change from baseline in morning pre-dose trough $\mathrm{FEV}_{1}$ over Weeks 12-24 in the global per-protocol (LSM difference $-11 \mathrm{~mL}, 95 \% \mathrm{CI}-39,17 \mathrm{~mL}$ ) and $\mathrm{mITT}$ populations (LSM difference $-15 \mathrm{~mL}, 95 \% \mathrm{CI}-42,12 \mathrm{~mL}$ ), using a non-inferiority margin of $-50 \mathrm{~mL}$ for the lower bound of the $95 \%$ CI. Similar treatment differences for BGF MDI vs. GFF MDI and BFF MDI were observed in both the Japanese and global populations for the secondary endpoint of change from baseline in morning pre-dose trough $\mathrm{FEV}_{1}$ over 24 weeks (Table 2, Figure 2).

While post-dose lung function assessments were not performed in the Japanese population, the secondary endpoints of $\mathrm{FEV}_{1} \mathrm{AUC}_{0-4}$, and peak change from baseline in $\mathrm{FEV}_{1}$ within 4 hrs post-dosing over Weeks 12-24 were significantly larger for BGF MDI vs. BFF MDI (both $P<0.0001$ ) but not vs. GFF MDI in the global population Supplementary Table 1). For $\mathrm{FEV}_{1} \mathrm{AUC}_{0-4}$, BFF MDI was non-inferior to BUD/FORM DPI using a margin of $-75 \mathrm{~mL}$ in the global per-protocol (LSM difference $-13 \mathrm{~mL}, 95 \% \mathrm{CI}-50,24 \mathrm{~mL}$ ), and $\mathrm{mITT}$ (LSM difference $-16 \mathrm{~mL}, 95 \% \mathrm{CI}-52,20 \mathrm{~mL}$ ) populations.

\section{Symptoms and Quality of Life Endpoints}

In the PDF version of the article, the units are broken over the line from the figures. Please ensure that these are kept together.Overall, changes from baseline in symptoms and quality of life endpoints were smaller in the Japanese vs. the global population (Table 3). This reflected less severe symptom burden at baseline in Japanese patients, as evidenced by higher Baseline Dyspnea Index (BDI) scores, lower SGRQ and CAT total scores, and lower use of rescue medication compared to the overall population. ${ }^{15}$ In the Japanese population, treatment with BGF MDI numerically improved TDI focal scores over Weeks 12-24 vs. GFF MDI and BFF MDI (LSM differences 0.17 [95\% CI $-0.17,0.50]$ and 0.22 [95\% CI -0.18 , 0.63], respectively; $P \leq 0.3279$; Table 3 ). Slightly smaller treatment differences in TDI focal score favoring BGF MDI vs. GFF MDI and BFF MDI were also seen in the global population (Table 3).

BGF MDI numerically improved SGRQ total score over Weeks $12-24$ vs. GFF MDI in both the Japanese (LSM difference $-1.52,95 \% \mathrm{CI}-3.60,0.55 ; P=0.1495)$ and global (LSM difference $-1.10,95 \% \mathrm{CI}-2.29,0.10 ; P=0.0717$ ) populations (Table 3). A small numerical improvement for BGF MDI vs. BFF MDI in SGRQ total score was observed in the global population (LSM difference $-0.52,95 \%$ CI $-1.99,0.95$; 
Table I Demographic and Baseline Disease Characteristics (Japanese Safety Population)

\begin{tabular}{|c|c|c|c|c|}
\hline & $\begin{array}{l}\text { BGF MDI } \\
320 / / 8 / 9.6 \mu g ~ N=139\end{array}$ & $\begin{array}{l}\text { GFF MDI } \\
\text { | } 8 / 9.6 \mu g ~ N=\mid 38\end{array}$ & $\begin{array}{l}\text { BFF MDI } \\
320 / 9.6 \mu \mathrm{gg} \mathrm{N}=70\end{array}$ & $\begin{array}{l}\text { BUD/FORM DPI } \\
400 / 12 \mu g ~ N=69\end{array}$ \\
\hline Mean age, years (SD) & $69.4(7.1)$ & $69.0(6.1)$ & $69.7(6.1)$ & 70.1 (6.8) \\
\hline Male, n (\%) & $130(93.5)$ & $126(91.3)$ & $68(97.1)$ & $68(98.6)$ \\
\hline Mean body mass index, $\mathrm{kg} / \mathrm{m}^{2}(\mathrm{SD})$ & $22.7(3.5)$ & $22.5(3.3)$ & $23.1(3.6)$ & $23.0(2.9)$ \\
\hline Current smoker, n (\%) & $40(28.8)$ & $41(29.7)$ & $19(27.1)$ & $21(30.4)$ \\
\hline Median number of pack-years smoked ${ }^{a}$ (range) & $51.0(10.0-186.1)$ & $52.0(10.0-135.0)$ & $50.0(11.5-192.0)$ & $55.0(12.5-180.0)$ \\
\hline \multicolumn{5}{|l|}{ COPD severity, n (\%) } \\
\hline Moderate & $72(5 \mid .8)$ & $74(53.6)$ & $38(54.3)$ & $37(53.6)$ \\
\hline Severe & $54(38.8)$ & $60(43.5)$ & $29(41.4)$ & $28(40.6)$ \\
\hline Very severe & $13(9.4)$ & $4(2.9)$ & $3(4.3)$ & $4(5.8)$ \\
\hline Mean COPD duration, years (SD) & $5.4(3.9)$ & $5.2(4.4)$ & $5.6(5.0)$ & $5.2(3.7)$ \\
\hline \multirow{2}{*}{\multicolumn{5}{|c|}{$\begin{array}{l}\text { Moderate or severe COPD exacerbations in the past } \\
12 \text { months, } \mathrm{n}(\%)\end{array}$}} \\
\hline & & & & \\
\hline 0 & $103(74.1)$ & $114(82.6)$ & $58(82.9)$ & $58(84.1)$ \\
\hline I & $25(18.0)$ & $16(11.6)$ & $10(14.3)$ & $6(8.7)$ \\
\hline$\geq 2$ & II (7.9) & $8(5.8)$ & $2(2.9)$ & $5(7.2)$ \\
\hline \multicolumn{5}{|l|}{ Moderate/severe exacerbation rate } \\
\hline Mean (SD) & $0.4(0.8)$ & $0.2(0.6)$ & $0.2(0.5)$ & $0.2(0.6)$ \\
\hline Median (range) & $0(0-5)$ & $0(0-4)$ & $0(0-3)$ & $0(0-2)$ \\
\hline \multicolumn{5}{|l|}{ Eosinophils } \\
\hline Median cells $/ \mathrm{mm}^{3}$ (range) & $150.0(30-670)$ & $162.5(25-2465)$ & $140.0(30-920)$ & $165.0(40-1040)$ \\
\hline$\geq 150$ cells $/ \mathrm{mm}^{3}, \mathrm{n}(\%)$ & $70(50.4)$ & $75(54.3)$ & $34(48.6)$ & $39(56.5)$ \\
\hline$\geq 300$ cells $/ \mathrm{mm}^{3}, \mathrm{n}(\%)$ & $18(13.0)$ & $22(15.9)$ & $8(11.4)$ & $7(10.1)$ \\
\hline Mean post-albuterol FEV,$\%$ predicted (SD) & $51.1(14.4)$ & $52.2(13.1)$ & $52.2(14.8)$ & $52.7(14.5)$ \\
\hline \multicolumn{5}{|l|}{ Reversibility to albuterol } \\
\hline Mean post-albuterol FEV & $179.9(\mid 17.0)$ & I74.I (I20.3) & $170.5(147.9)$ & 162.1 (119.4) \\
\hline - pre-albuterol $\mathrm{FEV}_{\mathrm{l}}, \mathrm{mL}(\mathrm{SD})$ & & & & \\
\hline Reversible ${ }^{\mathrm{b}}$, n (\%) & $55(39.6)$ & $51(37.0)$ & $24(34.3)$ & $26(37.7)$ \\
\hline Use of ICS at screening, n (\%) & $42(30.2)$ & $46(33.3)$ & $21(30.0)$ & $22(31.9)$ \\
\hline \multicolumn{5}{|l|}{ BDI focal score } \\
\hline $\mathrm{N}$ & 133 & 126 & 64 & 64 \\
\hline Mean (SD) & $7.5(1.8)$ & $7.5(1.8)$ & $7.7(2.1)$ & $7.6(1.8)$ \\
\hline \multicolumn{5}{|l|}{ SGRQ total score } \\
\hline $\mathrm{N}$ & 133 & 126 & 65 & 64 \\
\hline Mean (SD) & $35.6(14.4)$ & $37.0(13.8)$ & $32.7(13.7)$ & $34.2(14.7)$ \\
\hline Mean CAT total score (SD) & I5.4 (4.9) & $15.4(4.6)$ & $14.3(4.0)$ & $13.9(3.9)$ \\
\hline \multicolumn{5}{|l|}{ Rescue medication use ${ }^{c}$} \\
\hline $\mathrm{N}$ & 139 & 138 & 70 & 68 \\
\hline Mean (SD) & $0.8(1.3)$ & $0.7(1.7)$ & $0.5(1.2)$ & $0.5(1.4)$ \\
\hline Median (range), puffs/day & $0.0(0.0-6.6)$ & $0.0(0.0-12.1)$ & $0.0(0.0-6.9)$ & $0.0(0.0-8.0)$ \\
\hline
\end{tabular}

Notes: ${ }^{a}$ Number of pack-years smoked $=$ (number of cigarettes each day/20) $x$ number of years smoked. ${ }^{b}$ Reversible is defined as improvement in FEV 1 post-albuterol administration compared with pre-albuterol of $\geq 12 \%$ and $\geq 200 \mathrm{~mL}$. ${ }^{\mathrm{A}}$ Assessed during the last seven days of the screening period.

Abbreviations: BDI, Baseline Dyspnea Index; BFF, budesonide/formoterol fumarate; BGF, budesonide/glycopyrrolate/formoterol fumarate; BUD/FORM DPI, budesonide/ formoterol fumarate dry powder inhaler; CAT, COPD Assessment Test; COPD, chronic obstructive pulmonary disease; FEV, , forced expiratory volume in I s; GFF, glycopyrrolate/formoterol fumarate; ICS, inhaled corticosteroid; MDI, metered dose inhaler; SD, standard deviation; SGRQ, St George's Respiratory Questionnaire. 
Table 2 Primary and Secondary Lung Function Endpoints (Efficacy Estimand; mITT Population)

\begin{tabular}{|c|c|c|c|c|}
\hline & $\begin{array}{l}\text { BGF MDI } \\
320 / / 8 / 9.6 \mu g\end{array}$ & $\begin{array}{l}\text { GFF MDI } \\
\text { I 8/9.6 } \mu \mathrm{g}\end{array}$ & $\begin{array}{l}\text { BFF MDI } \\
320 / 9.6 \mu g\end{array}$ & $\begin{array}{l}\text { BUD/FORM DPI } \\
400 / / 2 \mu \mathrm{g}\end{array}$ \\
\hline \multicolumn{5}{|l|}{ Primary Endpoint } \\
\hline \multicolumn{5}{|c|}{ Change from baseline in morning pre-dose trough FEV (Weeks I2-24), mL } \\
\hline \multicolumn{5}{|l|}{ Japan } \\
\hline $\mathrm{n}$ & 134 & 126 & 65 & 65 \\
\hline LSM (SE) & $123(12.6)$ & $85(12.8)$ & $56(17.7)$ & $87(17.6)$ \\
\hline \multicolumn{5}{|l|}{ Treatment difference for BGF MDI vs. comparators } \\
\hline $\operatorname{LSM}(95 \% \mathrm{Cl})$ & NA & $37(3,72)$ & $67(25,109)$ & $35(-7,77)$ \\
\hline$P$-value & & 0.0337 & 0.0020 & 0.0996 \\
\hline \multicolumn{5}{|l|}{ Global } \\
\hline $\mathrm{n}$ & 592 & 559 & 278 & 288 \\
\hline LSM (SE) & $138(7.0)$ & $118(7.1)$ & $61(9.9)$ & $76(9.8)$ \\
\hline \multicolumn{5}{|l|}{ Treatment difference for BGF MDI vs. comparators } \\
\hline LSM $(95 \% \mathrm{Cl})$ & NA & $20(1,39)$ & $77(53,100)$ & $62(38,85)$ \\
\hline$P$-value & & 0.0424 & $<0.0001$ & $<0.0001$ \\
\hline \multicolumn{5}{|l|}{ Secondary Endpoint } \\
\hline \multicolumn{5}{|c|}{ Change from baseline in morning pre-dose trough FEV (over 24 weeks), mL } \\
\hline \multicolumn{5}{|l|}{ Japan } \\
\hline $\mathrm{n}$ & 137 & 134 & 68 & 68 \\
\hline LSM (SE) & $130(11.7)$ & $96(11.9)$ & $68(16.4)$ & $97(16.4)$ \\
\hline \multicolumn{5}{|l|}{ Treatment difference for BGF MDI vs. comparators } \\
\hline $\operatorname{LSM}(95 \% \mathrm{Cl})$ & NA & $34(2,66)$ & $62(23,101)$ & $33(-6,72)$ \\
\hline$P$-value & & 0.0368 & 0.0020 & 0.1014 \\
\hline \multicolumn{5}{|l|}{ Global $^{\mathrm{a}}$} \\
\hline $\mathrm{n}$ & 622 & 601 & 300 & 301 \\
\hline LSM (SE) & $147(6.5)$ & $125(6.6)$ & $73(9.2)$ & $88(9.1)$ \\
\hline \multicolumn{5}{|l|}{ Treatment difference for BGF MDI vs. comparators } \\
\hline LSM (95\% Cl) & NA & $22(4,39)$ & $74(52,95)$ & $59(38,80)$ \\
\hline$P$-value & & 0.0139 & $<0.0001$ & $<0.0001$ \\
\hline
\end{tabular}

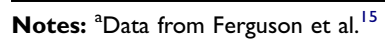

Abbreviations: BFF, budesonide/formoterol fumarate; BGF, budesonide/glycopyrrolate/formoterol fumarate; BUD/FORM DPI, budesonide/formoterol fumarate dry powder inhaler; $\mathrm{Cl}$, confidence interval; FEV , forced expiratory volume in I s; GFF, glycopyrrolate/formoterol fumarate; LSM, least squares mean; MDI, metered dose inhaler; mITT, modified intent-to-treat; NA, not applicable; SE, standard error.

$P=0.4893$ ) but not in the Japanese population (LSM difference $0.20,95 \%$ CI $-2.33,2.73 ; P=0.8766$; Table 3$)$.

BGF MDI numerically decreased E-RS total scores in Japanese patients (indicating an improvement in symptoms) vs. GFF MDI (LSM difference -0.89, 95\% CI $-1.86,0.07 ; P=0.0701$ ) and vs. BFF MDI (LSM difference $-0.52,95 \%$ CI $-1.70,0.66 ; \quad P=0.3899)$. There were also small numerical differences in E-RS scores in favor of BGF MDI vs. both dual comparators in the global population (Table 3).
Changes from baseline in daily rescue medication use over 24 weeks were small and similar across treatment groups in both the Japanese (range: 0.0 to -0.3 puffs/day) and global (range: -0.4 to $-0.7 \mathrm{puffs} /$ day) populations $(P \leq 0.4439$; Table 3$)$.

\section{CID and COPD Exacerbations}

The percentage of Japanese patients experiencing a CID was comparable across the BGF MDI, GFF MDI, and BFF MDI groups (range: 66-69\%), and slightly higher in the BUD/ 


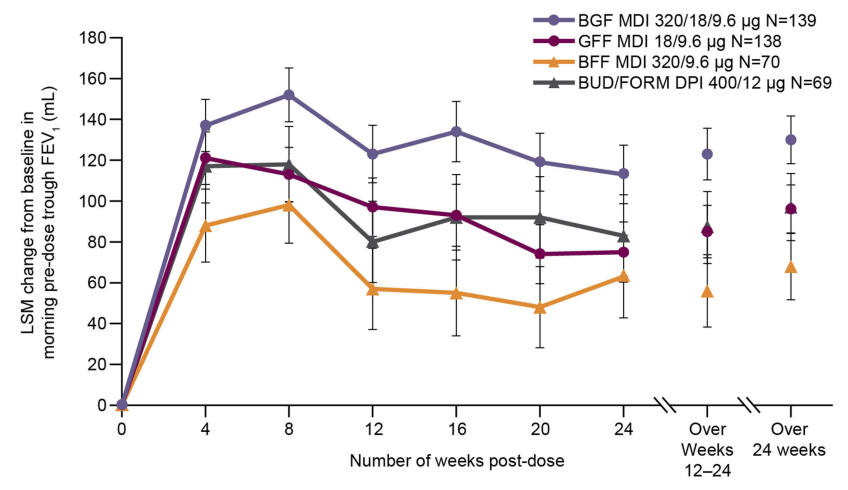

Figure 2 Change from baseline in morning pre-dose trough FEV , over 24 weeks (efficacy estimand; Japanese mITT population).

Note: Error bars represent standard error.

Abbreviations: BFF, budesonide/formoterol fumarate; BGF, budesonide/glycopyrrolate/ formoterol fumarate; BUD/FORM DPI, budesonide/formoterol fumarate dry powder inhaler; $\mathrm{FEV}_{\mathrm{l}}$, forced expiratory volume in I s; GFF, glycopyrrolate/formoterol fumarate; LSM, least squares mean; MDI, metered dose inhaler; mITT, modified intent-to-treat

FORM DPI group (74\%; Table 4). Hazard ratios for time to CID were $0.93,0.89$, and 0.81 for BGF MDI vs. GFF MDI, BFF MDI, and BUD/FORM DPI, respectively (Table 4).
Annualized rates of moderate or severe COPD exacerbations in Japanese patients over 24 weeks were $0.32,0.82,0.23$, and 0.28 for BGF MDI, GFF MDI, BFF MDI, and BUD/FORM DPI, respectively (Table 4). BGF MDI nominally significantly reduced the rate of moderate or severe exacerbations vs. GFF MDI (rate ratio $0.40,95 \%$ CI $0.19,0.83 ; P=0.0142$ ) but not vs. BFF MDI $(1.39,95 \%$ CI $0.47,4.13 ; P=0.5556)$. However, the number of subjects with exacerbations in the BFF MDI and BUD/FORM DPI groups was small (Table 4), and therefore no definitive conclusions can be derived from these comparisons. In a post hoc analysis of COPD exacerbations by reversibility at baseline, the lower rate of moderate or severe exacerbations for BGF MDI compared to GFF MDI was observed in both reversible (model-estimated rate 0.25 vs. 0.96 per year, respectively) and non-reversible ( 0.32 vs. 0.71 , respectively) patients in the Japanese population.

Table 3 Symptom and Quality of Life Endpoints (Efficacy Estimand; mITT Population)

\begin{tabular}{|c|c|c|c|c|}
\hline & $\begin{array}{l}\text { BGF MDI } \\
320 / 18 / 9.6 \mu g\end{array}$ & $\begin{array}{l}\text { GFF MDI } \\
\text { I8/9.6 } \mu \mathrm{g}\end{array}$ & $\begin{array}{l}\text { BFF MDI } \\
320 / 9.6 \mu g\end{array}$ & $\begin{array}{l}\text { BUD/FORM DPI } \\
400 / 12 \mu \mathrm{g}\end{array}$ \\
\hline \multicolumn{5}{|l|}{ TDI focal score over Weeks I2-24 } \\
\hline \multicolumn{5}{|l|}{ Japan } \\
\hline $\mathrm{n}$ & 133 & 126 & 64 & 64 \\
\hline LSM (SE) & $0.42(0.12)$ & $0.25(0.12)$ & $0.19(0.17)$ & $0.07(0.17)$ \\
\hline \multicolumn{5}{|l|}{ Treatment difference for BGF MDI vs. comparators } \\
\hline $\operatorname{LSM}(95 \% \mathrm{Cl})$ & NA & $0.17(-0.17,0.50)$ & $0.22(-0.18,0.63)$ & $0.34(-0.06,0.75)$ \\
\hline$P$-value & & 0.3279 & 0.2804 & 0.0952 \\
\hline \multicolumn{5}{|l|}{ Global } \\
\hline $\mathrm{n}$ & 585 & 544 & 274 & 280 \\
\hline LSM (SE) & $1.21(0.10)$ & $1.13(0.10)$ & $1.06(0.14)$ & $0.72(0.14)$ \\
\hline \multicolumn{5}{|l|}{ Treatment difference for BGF MDI vs. comparators } \\
\hline LSM (95\% Cl) & NA & $0.07(-0.20,0.35)$ & $0.15(-0.18,0.48)$ & $0.49(0.16,0.82)$ \\
\hline$P$-value & & 0.5928 & 0.3729 & 0.0037 \\
\hline \multicolumn{5}{|c|}{ Change from baseline in SGRQ total score over Weeks I2-24 } \\
\hline \multicolumn{5}{|l|}{ Japan } \\
\hline $\mathrm{n}$ & 133 & 126 & 65 & 64 \\
\hline LSM (SE) & $-4.1(0.75)$ & $-2.6(0.77)$ & $-4.3(1.06)$ & $-3.4(1.06)$ \\
\hline \multicolumn{5}{|l|}{ Treatment difference for BGF MDI vs. comparators } \\
\hline LSM (95\% Cl) & NA & $-1.52(-3.60,0.55)$ & $0.20(-2.33,2.73)$ & $-0.73(-3.26,1.81)$ \\
\hline$P$-value & & 0.1495 & 0.8766 & 0.5724 \\
\hline \multicolumn{5}{|l|}{ Global } \\
\hline $\mathrm{n}$ & 592 & 553 & 277 & 284 \\
\hline LSM (SE) & $-7.9(0.50)$ & $-6.8(0.51)$ & $-7.4(0.67)$ & $-6.5(0.67)$ \\
\hline
\end{tabular}

(Continued) 
Table 3 (Continued).

\begin{tabular}{|c|c|c|c|c|}
\hline & $\begin{array}{l}\text { BGF MDI } \\
320 / / 8 / 9.6 \mu \mathrm{g}\end{array}$ & $\begin{array}{l}\text { GFF MDI } \\
\text { I 8/9.6 } \mu \mathrm{g}\end{array}$ & $\begin{array}{l}\text { BFF MDI } \\
320 / 9.6 \mu g\end{array}$ & $\begin{array}{l}\text { BUD/FORM DPI } \\
400 / 12 \mu \mathrm{g}\end{array}$ \\
\hline $\begin{array}{l}\text { Treatment difference for BGF MDI vs. comparators } \\
\text { LSM }(95 \% \mathrm{Cl}) \\
P \text {-value }\end{array}$ & NA & $\begin{array}{l}-1.10(-2.29,0.10) \\
0.0717\end{array}$ & $\begin{array}{l}-0.52(-1.99,0.95) \\
0.4893\end{array}$ & $\begin{array}{l}-1.42(-2.88,0.04) \\
0.0570\end{array}$ \\
\hline \multicolumn{5}{|c|}{ Change from baseline in E-RS total score over Weeks I2-24 } \\
\hline $\begin{array}{l}\text { Japan } \\
\qquad \mathrm{n} \\
\text { LSM (SE) }\end{array}$ & $\begin{array}{l}134 \\
-0.9(0.35)\end{array}$ & $\begin{array}{l}127 \\
0.0(0.35)\end{array}$ & $\begin{array}{l}65 \\
-0.4(0.49)\end{array}$ & $\begin{array}{l}66 \\
-0.3(0.49)\end{array}$ \\
\hline $\begin{array}{l}\text { Treatment difference for BGF MDI vs. comparators } \\
\text { LSM }(95 \% \mathrm{Cl}) \\
P \text {-value }\end{array}$ & NA & $\begin{array}{l}-0.89(-1.86,0.07) \\
0.0701\end{array}$ & $\begin{array}{l}-0.52(-1.70,0.66) \\
0.3899\end{array}$ & $\begin{array}{l}-0.60(-1.78,0.58) \\
0.3204\end{array}$ \\
\hline $\begin{array}{l}\text { Global } \\
\qquad \mathrm{n} \\
\text { LSM (SE) }\end{array}$ & $\begin{array}{l}596 \\
-1.1(0.16)\end{array}$ & $\begin{array}{l}560 \\
-0.8(0.16)\end{array}$ & $\begin{array}{l}278 \\
-1.0(0.23)\end{array}$ & $\begin{array}{l}292 \\
-0.9(0.22)\end{array}$ \\
\hline $\begin{array}{l}\text { Treatment difference for BGF MDI vs. comparators } \\
\text { LSM }(95 \% \mathrm{Cl}) \\
P \text {-value }\end{array}$ & NA & $\begin{array}{l}-0.29(-0.72,0.15) \\
0.1965\end{array}$ & $\begin{array}{l}-0.05(-0.59,0.48) \\
0.8445\end{array}$ & $\begin{array}{l}-0.16(-0.69,0.37) \\
0.5538\end{array}$ \\
\hline Change from baseline in average daily rescue & tion use over 2 & puffs/day & & \\
\hline $\begin{array}{l}\text { Japan } \\
\qquad \mathrm{n} \\
\text { LSM (SE) }\end{array}$ & $\begin{array}{l}139 \\
-0.2(0.07)\end{array}$ & $\begin{array}{l}138 \\
0.0(0.07)\end{array}$ & $\begin{array}{l}70 \\
-0.1(0.10)\end{array}$ & $\begin{array}{l}68 \\
-0.3(0.10)\end{array}$ \\
\hline $\begin{array}{l}\text { Treatment difference for BGF MDI vs. comparators } \\
\text { LSM }(95 \% \mathrm{Cl}) \\
P \text {-value }\end{array}$ & NA & $\begin{array}{l}-0.13(-0.32,0.06) \\
0.1831\end{array}$ & $\begin{array}{l}-0.09(-0.33,0.14) \\
0.4439\end{array}$ & $\begin{array}{l}0.12(-0.12,0.35) \\
0.3358\end{array}$ \\
\hline $\begin{array}{l}\text { Global } \\
\text { n } \\
\text { LSM (SE) }\end{array}$ & $\begin{array}{l}638 \\
-0.5(0.06)\end{array}$ & $\begin{array}{l}621 \\
-0.4(0.06)\end{array}$ & $\begin{array}{l}313 \\
-0.4(0.08)\end{array}$ & $\begin{array}{l}313 \\
-0.7(0.08)\end{array}$ \\
\hline $\begin{array}{l}\text { Treatment difference for BGF MDI vs. comparators } \\
\text { LSM }(95 \% \mathrm{Cl}) \\
P \text {-value }\end{array}$ & NA & $\begin{array}{l}-0.12(-0.28,0.04) \\
0.1543\end{array}$ & $\begin{array}{l}-0.14(-0.34,0.06) \\
0.1793\end{array}$ & $\begin{array}{l}0.16(-0.04,0.36) \\
0.1198\end{array}$ \\
\hline
\end{tabular}

Abbreviations: BFF, budesonide/formoterol fumarate; BGF, budesonide/glycopyrrolate/formoterol fumarate; BUD/FORM DPI, budesonide/formoterol fumarate dry powder inhaler; $\mathrm{Cl}$, confidence interval; COPD, chronic obstructive pulmonary disease; E-RS, Evaluating Respiratory Symptoms in COPD Total score; GFF, glycopyrrolate/formoterol fumarate; LSM, least squares mean; MDI, metered dose inhaler; mITT, modified intent-to-treat; NA, not applicable; SE, standard error; SGRQ, St George's Respiratory Questionnaire; TDI, Transition Dyspnea Index.

\section{Safety}

The percentage of Japanese patients who experienced at least one treatment-emergent adverse event (TEAE) ranged from $59.4 \%$ in the BUD/FORM DPI group to $72.9 \%$ in the BFF MDI group (Table 5). Overall, the most commonly observed TEAEs in Japanese patients were nasopharyngitis (19.7\%) and bronchitis $(5.3 \%)$. The incidence of serious TEAEs was comparable across treatment groups (range: 7.9-10.1\%; Table 5); the most frequent serious TEAEs in the Japanese population overall were COPD (2.9\%) and pneumonia (1.0\%). Eight patients in the Japan subgroup (1.9\%) had confirmed pneumonia events, seven of which occurred in the BGF MDI group (5.0\%); the incidence of confirmed pneumonia ranged from $0 \%$ to $0.7 \%$ across the other three treatment groups. Of the seven confirmed cases of pneumonia in the BGF MDI, only one was considered by the investigator to be treatment-related. A total of three Japanese patients $(0.7 \%)$ had an event confirmed as MACE: two cases of non-fatal myocardial infarction (both in the BUD/FORM DPI group), and one cardiovascular death in the GFF MDI group. Neither of the two deaths in the Japan subgroup that resulted from TEAEs reported 
Table $4 \mathrm{CID}^{\mathrm{a}}$ and COPD Exacerbations (Efficacy Estimand; Japanese mITT Population)

\begin{tabular}{|c|c|c|c|c|}
\hline & $\begin{array}{l}\text { BGF MDI } \\
320 / / 8 / 9.6 \mu g N=139\end{array}$ & $\begin{array}{l}\text { GFF MDI } \\
\text { I } 8 / 9.6 \mu g ~ N=\mid 38\end{array}$ & $\begin{array}{l}\text { BFF MDI } \\
320 / 9.6 \mu g ~ N=70\end{array}$ & $\begin{array}{l}\text { BUD/FORM DPI } \\
400 / / 2 \mu \mathrm{g} N=69\end{array}$ \\
\hline \multicolumn{5}{|l|}{ Time to $\mathrm{CID}^{\mathrm{a}}$} \\
\hline Patients with CID, n (\%) & $91(65.5)$ & $92(66.7)$ & $48(68.6)$ & 51 (73.9) \\
\hline $\begin{array}{l}\text { Treatment difference for BGF MDI vs. comparators } \\
\text { Hazard ratio }(95 \% \mathrm{Cl}) \\
P \text {-value }\end{array}$ & NA & $\begin{array}{l}0.93(0.70,1.25) \\
0.6461\end{array}$ & $\begin{array}{l}0.89(0.63,1.27) \\
0.5348\end{array}$ & $\begin{array}{l}0.81(0.57, \mathrm{I} .14) \\
0.2270\end{array}$ \\
\hline \multicolumn{5}{|c|}{ Rate of moderate or severe COPD exacerbations, model-estimated rate per year } \\
\hline $\begin{array}{l}\text { Patients with exacerbations, } \mathrm{n}(\%) \\
\text { Rate }\end{array}$ & $\begin{array}{l}20(14.4) \\
0.32\end{array}$ & $\begin{array}{l}28(20.3) \\
0.82\end{array}$ & $\begin{array}{l}7(10.0) \\
0.23\end{array}$ & $\begin{array}{l}9(13.0) \\
0.28\end{array}$ \\
\hline $\begin{array}{l}\text { Treatment difference for BGF MDI vs. comparators } \\
\text { Rate ratio }(95 \% \mathrm{Cl}) \\
\text { P-value }\end{array}$ & NA & $\begin{array}{l}0.40(0.19,0.83) \\
0.0142\end{array}$ & $\begin{array}{l}1.39(0.47,4.13) \\
0.5556\end{array}$ & $\begin{array}{l}1.16(0.43,3.16) \\
0.7644\end{array}$ \\
\hline
\end{tabular}

Notes: ${ }^{\mathrm{a}} \mathrm{CID}$ was defined as a $\geq 100 \mathrm{~mL}$ decrease from baseline in trough $\mathrm{FEV}$, ; a $\geq 4$ point increase from baseline in SGRQ total score; a TDI focal score of -I point or less; or a treatment-emergent moderate or severe COPD exacerbation occurring up to Week 24.

Abbreviations: BFF, budesonide/formoterol fumarate; BGF, budesonide/glycopyrrolate/formoterol fumarate; BUD/FORM DPI, budesonide/formoterol fumarate dry powder inhaler; $\mathrm{Cl}$, confidence interval; $\mathrm{CID}$, clinically important deterioration; COPD, chronic obstructive pulmonary disease; FEV, forced expiratory volume in I s; GFF, glycopyrrolate/formoterol fumarate; MDI, metered dose inhaler; mITT, modified intent-to-treat; NA, not applicable; SGRQ, St George's Respiratory Questionnaire; TDI, Transition Dyspnea Index.

during the study were considered by the investigator to be related to study treatment (one each in the GFF MDI and BFF MDI groups resulting from cardio-respiratory arrest and squamous cell carcinoma, respectively). No clinically meaningful trends in clinical laboratory values, vital signs, or electrocardiogram parameters were observed in any of the treatment groups.

\section{Discussion}

The KRONOS study provided support for the use of triple therapy in a global population of symptomatic patients with COPD (CAT score $\geq 10$ despite treatment with two or more inhaled maintenance therapies), the majority of whom had not experienced an exacerbation in the past year. ${ }^{15}$ Here, we present findings from a pre-specified analysis of the Japan subgroup of KRONOS, demonstrating that triple therapy improved lung function compared to both dual therapies in Japanese patients with COPD and also reduced exacerbations compared to LAMA/LABA therapy.

Overall, efficacy and safety findings were comparable between the Japanese and global populations, with the degree of variation expected given the smaller sample size of the Japan subgroup, especially in the BFF MDI and BUD/ FORM DPI groups due to the 2:2:1:1 randomization ratio. While both the global and Japanese populations were similar in terms of airflow limitation, exacerbation history, and eosinophil count, the Japanese population was slightly older on average and had slightly higher mean BDI focal scores and lower SGRQ and CAT total scores at baseline, indicating a slightly lower symptom burden overall. ${ }^{15}$ In addition, use of ICS at baseline was lower in Japanese patients relative to the global population. These demographic differences are generally consistent with the results of other subgroup analyses of Japanese patients with COPD who participated in similar global Phase III clinical studies of LAMA/LABA therapies. ${ }^{10,21}$

For morning pre-dose trough $\mathrm{FEV}_{1}$, treatment comparisons showed larger improvements with BGF MDI compared to GFF MDI and BFF MDI, which were generally consistent between the Japan and global populations. BGF MDI also showed numerical benefits on symptoms endpoints vs. GFF MDI and BFF MDI in Japanese patients, with treatment differences similar to the global population. The variability in the magnitude of improvement from baseline in the Japanese population compared to the global population was consistent with the smaller sample size and the fact that Japanese patients reported less severe symptoms and higher quality of life at baseline compared to the overall patient population. ${ }^{15}$

All three ICS-containing treatments showed a marked effect on exacerbation rates vs. LAMA/LABA therapy in Japanese patients. This is notable as the current JRS 
Table 5 Summary of Adverse Events (Japanese Safety Population)

\begin{tabular}{|c|c|c|c|c|}
\hline & $\begin{array}{l}\text { BGF MDI } \\
320 / / 8 / 9.6 \mu g ~ N=139\end{array}$ & $\begin{array}{l}\text { GFF MDI } \\
\text { | } 8 / 9.6 \mu g ~ N=\mid 38\end{array}$ & $\begin{array}{l}\text { BFF MDI } \\
320 / 9.6 \mu g ~ N=70\end{array}$ & $\begin{array}{l}\text { BUD/FORM DPI } \\
400 / / 2 \mu g ~ N=69\end{array}$ \\
\hline \multicolumn{5}{|l|}{ TEAEs, $\mathrm{n}(\%)$} \\
\hline Patients with $\geq I$ TEAE & $93(66.9)$ & $92(66.7)$ & 51 (72.9) & $4 I(59.4)$ \\
\hline Patients with TEAEs related ${ }^{\mathrm{a}}$ to study treatment & $29(20.9)$ & II (8.0) & $12(17.1)$ & $4(5.8)$ \\
\hline Patients with serious TEAEs & II (7.9) & $14(10.1)$ & $7(10.0)$ & $6(8.7)$ \\
\hline $\begin{array}{l}\text { Patients with serious TEAEs related }{ }^{\text {a }} \text { to study } \\
\text { treatment }\end{array}$ & $\mathrm{I}(0.7)$ & $4(2.9)$ & 0 & 0 \\
\hline $\begin{array}{l}\text { Patients with TEAEs that led to early } \\
\text { discontinuation }\end{array}$ & $6(4.3)$ & $6(4.3)$ & $2(2.9)$ & $2(2.9)$ \\
\hline Patients with confirmed ${ }^{\mathrm{b}} \mathrm{MACE}$ & 0 & I (0.7) & 0 & $2(2.9)$ \\
\hline Patients with confirmed $d^{\mathrm{b}, \mathrm{c}}$ pneumonia & $7(5.0)$ & $\mathrm{I}(0.7)$ & 0 & 0 \\
\hline Deaths (all causes) & 0 & I (0.7) & $\mathrm{I}(\mathrm{I} .4)$ & 0 \\
\hline \multicolumn{5}{|l|}{$\begin{array}{l}\text { TEAEs occurring in } \geq 3 \% \text { of patients in any treatment } \\
\text { arm, preferred term, } n(\%)\end{array}$} \\
\hline Nasopharyngitis & $29(20.9)$ & $23(16.7)$ & $14(20.0)$ & $16(23.2)$ \\
\hline Bronchitis & $6(4.3)$ & $6(4.3)$ & $7(10.0)$ & $3(4.3)$ \\
\hline Dysphonia & $9(6.5)$ & I (0.7) & $8(11.4)$ & $3(4.3)$ \\
\hline Muscle spasms & $12(8.6)$ & $2(1.4)$ & $5(7.1)$ & $2(2.9)$ \\
\hline Upper respiratory tract infection & $7(5.0)$ & $5(3.6)$ & $\mathrm{I}(\mathrm{I} .4)$ & $\mathrm{I}(\mathrm{I} .4)$ \\
\hline Upper respiratory tract inflammation & $4(2.9)$ & $6(4.3)$ & $3(4.3)$ & 0 \\
\hline COPD $^{d}$ & $4(2.9)$ & $5(3.6)$ & $2(2.9)$ & $\mathrm{I}(\mathrm{I} .4)$ \\
\hline Influenza & $\mathrm{I}(0.7)$ & $4(2.9)$ & I (I.4) & $4(5.8)$ \\
\hline Eczema & $\mathrm{I}(0.7)$ & $2(1.4)$ & $2(2.9)$ & $3(4.3)$ \\
\hline Pneumonia ${ }^{\mathrm{c}, \mathrm{e}}$ & $6(4.3)$ & $\mathrm{I}(0.7)$ & $\mathrm{I}(\mathrm{I} .4)$ & 0 \\
\hline
\end{tabular}

Notes: ${ }^{\mathrm{a} P o s s i b l y, ~ p r o b a b l y, ~ o r ~ d e f i n i t e l y ~ r e l a t e d ~ i n ~ t h e ~ o p i n i o n ~ o f ~ t h e ~ i n v e s t i g a t o r . ~}{ }^{\mathrm{b}}$ Confirmed by clinical endpoint committee. ${ }^{\mathrm{C} O n e}$ confirmed case of pneumonia in the BGF MDI group was reported under the preferred term of interstitial lung disease, and therefore was not categorized as a TEAE of pneumonia. ${ }^{d}$ Worsening of COPD. eSpontaneously reported as adverse events; not confirmed by clinical endpoint committee.

Abbreviations: BFF, budesonide/formoterol fumarate; BGF, budesonide/glycopyrrolate/formoterol fumarate; BUD/FORM DPI, budesonide/formoterol fumarate dry powder inhaler; COPD, chronic obstructive pulmonary disease; GFF, glycopyrrolate/formoterol fumarate; MACE, major adverse cardiovascular events; MDI, metered dose inhaler; TEAE, treatment-emergent adverse event.

guidelines only recommend ICS treatment for patients with evidence of ACO. ${ }^{6}$ However, patients with a current diagnosis of asthma were not permitted to enroll in KRONOS. Additionally, only a small proportion of the Japanese subpopulation showed characteristic features of $\mathrm{ACO},{ }^{7,8}$ with only $3.6 \%$ having reversibility to albuterol $\geq 400 \mathrm{~mL}$, and $13.2 \%$ having a blood eosinophil count $\geq 300$ cells $/ \mathrm{mm}^{3}$ at baseline. Moreover, the exacerbation findings were consistent across both reversible and non-reversible patients, suggesting that the response to ICS treatment in Japanese patients was not due to an asthma component. While no meaningful differences in exacerbation rates were observed between the triple and dual ICS-containing therapies in the Japan subgroup (in contrast to the numerical improvement seen with BGF MDI vs. BFF MDI in the global population), this may be a result of baseline differences in prior exacerbation history between the BGF MDI group and both ICS/ LABA groups as well as the smaller sample size of the Japan population. Indeed, the small sample size and the relatively low number of exacerbation events in Japanese patients resulted in an estimate with high variability, and thus these findings should be interpreted with caution. The slightly lower overall rate of exacerbations in Japanese patients compared to the global population is consistent with other Japanese and Asian subgroup analyses in COPD, ${ }^{9,10,22}$ as well as the fact that $80 \%$ of the Japanese population had no history of exacerbation in the previous year. The reduction in exacerbations observed with ICS-containing therapies compared to GFF MDI suggests that many symptomatic patients in Japan could benefit from the addition of an ICS to their treatment regimen (regardless of exacerbation history), as only $31.5 \%$ were receiving ICS therapy at study entry (compared to $71.8 \%$ of the global population).

Overall, all treatments were generally well tolerated in Japanese patients, and the safety profile of BGF MDI was comparable to the results from the global population. These 
findings are in agreement with a previous Phase I study of BGF MDI, which found that exposure and tolerability were comparable between Western and Japanese healthy subjects. $^{23}$ Although some numerical differences were observed in the adverse event profile between the Japanese and global KRONOS populations, ${ }^{15}$ the differences were generally small in magnitude and to be expected, based on the smaller sample size of the Japanese subgroup. With regard to pneumonia, an important safety outcome with ICS-containing therapies in COPD, the highest incidence of confirmed pneumonia was observed in the BGF MDI group $(n=7 ; 5.0 \%)$, and the incidence was similar between the ICS/LABA and LAMA/LABA groups $(\mathrm{n}=0-1 ; 0-0.7 \%)$. This was most likely a chance finding based on several factors. Firstly, there was no clear trend for a higher incidence of pneumonia in the budesonide-containing treatment groups (BGF MDI, 5.0\%; BFF MDI 0\%; BUD/FORM DPI, 0\%) compared to GFF MDI (0.7\%). Secondly, there was an imbalance in the baseline exacerbation history across treatment groups that may have contributed to the imbalance in the incidence of pneumonia. Specifically, the percentage of patients who had experienced an exacerbation in the previous year was highest in the BGF MDI group (25.9\%) vs. all three dual therapy groups (range: 15.9-17.4\%), and a previous exacerbation is a risk factor for pneumonia in patients with COPD. ${ }^{24}$ Accordingly, five of the seven patients with confirmed pneumonia in the BGF MDI group had a history of exacerbation in the past 12 months, while the patient in the GFF MDI group who developed pneumonia did not have a prior history of exacerbation. Furthermore, the incidence of pneumonia in the global population was comparable for all treatments (range: $1.3-1.9 \%),{ }^{15}$ and overall rates of pneumonia across groups were comparable between the Japanese (1.9\%) and global $(1.7 \%)$ populations. Finally, in an extension of the current study, which evaluated Japanese patients enrolled in KRONOS for an additional 28 weeks, the incidence of pneumonia during Weeks 24-52 of treatment was similar across the BGF MDI (4.3\%) and dual therapy treatment groups (range: $2.9-5.7 \%$ ). ${ }^{25}$ Across the 52-week study, five of the 13 patients in the BGF MDI group with confirmed pneumonia (and none of the 11 patients in the other three treatment groups) had experienced an exacerbation in the previous year.

A limitation of the study is the small sample size in the Japanese subgroup, requiring the conclusions to be interpreted with caution; however, this factor is somewhat mitigated by the generally similar results between the global and Japanese populations.

\section{Conclusions}

BGF MDI nominally significantly improved lung function and numerically improved symptoms compared with LAMA/LABA (GFF MDI) and ICS/LABA (BFF MDI) combination therapies. BGF MDI also nominally significantly reduced the rate of moderate or severe COPD exacerbations vs. GFF MDI in Japanese patients with COPD despite the fact that these patients did not meet criteria for asthma-COPD overlap. Efficacy and safety findings were generally comparable to the global KRONOS study population. These results suggest that triple therapy with BGF MDI is efficacious and well tolerated in Japanese patients with moderate-to-very severe COPD, and may be a useful treatment option for those who experience continued symptoms on dual therapy, regardless of exacerbation history and bronchodilator reversibility.

\section{Abbreviations}

ACO, asthma-COPD overlap; AUC, area under the curve; BDI, Baseline Dyspnea Index; BFF, budesonide/formoterol fumarate; BGF, budesonide/glycopyrrolate/formoterol fumarate; BUD/FORM DPI, budesonide/formoterol dry powder inhaler; CEC, clinical endpoint committee; $\mathrm{CI}$, confidence interval; CID, clinically important deterioration; E-RS, Evaluating Respiratory Symptoms; FEV 1 , forced expiratory volume in $1 \mathrm{~s}$; GFF, glycopyrrolate/formoterol fumarate; GOLD, Global Initiative for Chronic Obstructive Lung Disease; ICS, inhaled corticosteroid; JRS, Japanese Respiratory Society; LABA, long-acting $\beta_{2}$-agonist; LAMA, long-acting muscarinic antagonist; LSM, least squares mean; MACE, major adverse cardiovascular events; MDI, metered dose inhaler; mITT, modified intent-to-treat; SGRQ, St George's Respiratory Questionnaire; TDI, Transition Dyspnea Index; TEAE, treatment-emergent adverse event.

\section{Ethics Approval and Informed Consent}

Patients provided written informed consent prior to screening, and the study was conducted in accordance with Good Clinical Practice, including the Declaration of Helsinki and the International Council for Harmonisation. The protocol was approved by local institutional review boards (names 
have been previously published). ${ }^{15}$ The names of all institutional review boards can be found in Supplementary Table 2 .

\section{Data Sharing Statement}

Data underlying the findings described in this manuscript may be obtained in accordance with AstraZeneca's data sharing policy described at: https://astrazenecagrouptrials. pharmacm.com/ST/Submission/Disclosure.

\section{Acknowledgments}

The authors would like to thank all the patients and their families and the team of investigators, research nurses, and operations staff involved in these studies. Medical writing support, under the direction of the authors, was provided by Julia King, $\mathrm{PhD}$, of CMC Connect, a division of McCann Health Medical Communications Ltd, Glasgow, UK and was funded by AstraZeneca, Gaithersburg, USA, in accordance with Good Publication Practice (GPP3) guidelines. ${ }^{26}$

\section{Author Contributions}

$\mathrm{HO}, \mathrm{MT}, \mathrm{EB}, \mathrm{SB}, \mathrm{KdA}, \mathrm{PD}$, and $\mathrm{CR}$ contributed to the conception or design of the study; MI, YF, YI, OH, GTR, $\mathrm{KFR}, \mathrm{HO}, \mathrm{MT}, \mathrm{EB}, \mathrm{KdA}$, and MA participated in the acquisition of reported data; $\mathrm{NH}$ and $\mathrm{SB}$ contributed to the statistical analysis of the data. All authors participated in the analysis and interpretation of data reported. All authors reviewed or critically revised the manuscript, provided final approval of the version to be published, and agreed to be accountable for all aspects of the work.

\section{Funding}

The KRONOS study was supported by AstraZeneca.

\section{Disclosure}

MI reports personal fees from AstraZeneca, during the conduct of the study; and personal fees from Kyorin, Nippon Boehringer Ingelheim, and Novartis Pharma, outside of the submitted work. YI reports personal fees from GlaxoSmithKline, Nippon Boehringer Ingelheim, Nobelpharma, and Shionogi \& Co., outside of the submitted work. $\mathrm{OH}$ reports personal fees from AstraZeneca, Nippon Boehringer Ingelheim, and Novartis Pharma; and research funding from AstraZeneca, Daiichi Sankyo, GlaxoSmithKline, Kyorin Pharm, Nippon Boehringer Ingelheim, Novartis Pharma, and Ono Pharm. GTF reports grants, personal fees, and non-financial support from AstraZeneca during the conduct of the study; grants, personal fees, and non-financial support from AstraZeneca, Boehringer
Ingelheim, Novartis, Pearl - a member of the AstraZeneca Group, and Sunovion; grants and personal fees from Theravance; and personal fees from Circassia, GlaxoSmithKline, Innoviva, Mylan, Sanofi, and Verona, outside of the submitted work. KFR reports personal fees from AstraZeneca, Berlin-Chemie, Boehringer Ingelheim, Chiesi Pharmaceuticals, InterMune, Novartis, Sanofi, Roche, and Teva; and grants from the Ministry of Education and Science, Germany, outside of the submitted work. NH, HO, and MT are employees of AstraZeneca K.K., Japan. EB, SB, MA, PD, and $\mathrm{CR}$ are employees of AstraZeneca. $\mathrm{KdA}$ is a former employee of AstraZeneca. The authors report no other conflicts of interest in this work.

\section{References}

1. Fukuchi Y, Nishimura M, Ichinose $M$, et al. COPD in Japan: the Nippon COPD epidemiology study. Respirology. 2004;9(4):458-465. doi:10.1111/res.2004.9.issue-4

2. Nomura S, Sakamoto H, Glenn S, et al. Population health and regional variations of disease burden in Japan, 1990-2015: a systematic subnational analysis for the Global Burden of Disease Study 2015. Lancet. 2017;390(10101):1521-1538. doi:10.1016/ S0140-6736(17)31544-1

3. Omori H, Yoshimoto D, Kumar M, Goren A. Prevalence, awareness, characteristics, and health outcomes associated with COPD at-risk status among adults in Japan. Expert Rev Pharmacoecon Outcomes Res. 2016;16(4):501-512. doi:10.1586/14737167.2016.1104250

4. The World Bank. Population ages 65 and above (\% of total); 2017. Available from: https://data.worldbank.org/indicator/SP.POP.65UP. TO.ZS. Accessed February 25, 2019.

5. Global Initiative for Chronic Obstructive Lung Disease. 2019 Report: global strategy for prevention, diagnosis and management of COPD; 2019. Available from: http://www.goldcopd.org. Accessed April 11, 2019.

6. The Japanese Respiratory Society. Guidelines for the diagnosis and treatment of COPD, 5th Edition (in Japanese); 2018. Available from: http:// www.jrs.or.jp/modules/guidelines/index.php?content_id=112. Accessed February 26, 2019.

7. Yanagisawa S, Ichinose M. Definition and diagnosis of asthma-COPD overlap (ACO). Allergol Int. 2018;67(2):172-178. doi:10.1016/j. alit.2018.01.002

8. Sin DD, Miravitlles M, Mannino DM, et al. What is asthma-COPD overlap syndrome? Towards a consensus definition from a round table discussion. Eur Respir J. 2016;48(3):664-673. doi:10.1183/13993003.00436-2016

9. Fukuchi Y, Fernandez L, Kuo HP, et al. Efficacy of tiotropium in COPD patients from Asia: a subgroup analysis from the UPLIFT trial. Respirology. 2011;16(5):825-835. doi:10.1111/j.14401843.2011.01982.x

10. Ichinose M, Taniguchi H, Takizawa A, et al. The efficacy and safety of combined tiotropium and olodaterol via the Respimat ${ }^{\mathbb{R}}$ inhaler in patients with COPD: results from the Japanese sub-population of the Tonado ${ }^{\circledR}$ studies. Int J Chron Obstruct Pulmon Dis. 2016;11:2017-2027. doi:10.2147/COPD.S110389

11. Lipson DA, Barnhart F, Brealey N, et al. Once-daily single-inhaler triple versus dual therapy in patients with COPD. $N$ Engl J Med. 2018;378(18):1671-1680. doi:10.1056/NEJMoa1713901

12. Papi A, Vestbo J, Fabbri L, et al. Extrafine inhaled triple therapy versus dual bronchodilator therapy in chronic obstructive pulmonary disease (TRIBUTE): a double-blind, parallel group, randomised controlled trial. Lancet. 2018;391(10125):1076-1084. doi:10.1016/ S0140-6736(18)30206-X 
13. Singh D, Papi A, Corradi M, et al. Single inhaler triple therapy versus inhaled corticosteroid plus long-acting $\beta_{2}$-agonist therapy for chronic obstructive pulmonary disease (TRILOGY): a double-blind, parallel group, randomised controlled trial. Lancet. 2016;388 (10048):963-973. doi:10.1016/S0140-6736(16)31354-X

14. Lipson DA, Barnacle H, Birk R, et al. FULFIL trial: once-daily triple therapy for patients with chronic obstructive pulmonary disease. Am $J$ Respir Crit Care Med. 2017;196(4):438-446. doi:10.1164/ rccm.201703-04490C

15. Ferguson GT, Rabe KF, Martinez FJ, et al. Triple therapy with budesonide/glycopyrrolate/formoterol fumarate with co-suspension delivery technology versus dual therapies in chronic obstructive pulmonary disease (KRONOS): a double-blind, parallel-group, multicentre, phase 3 randomised controlled trial. Lancet Respir Med. 2018;6(10):747-758. doi:10.1016/S2213-2600(18)30327-8

16. Takahashi S, Betsuyaku T. The chronic obstructive pulmonary disease comorbidity spectrum in Japan differs from that in western countries. Respir Investig. 2015;53(6):259-270. doi:10.1016/j. resinv.2015.05.005

17. Nishimura M. Similarities and differences between East and West in COPD. Respirology. 2016;21(8):1340-1341. doi:10.1111/ resp.2016.21.issue-8

18. Landis SH, Muellerova H, Mannino DM, et al. Continuing to confront COPD international patient survey: methods, COPD prevalence, and disease burden in 2012-2013. Int J Chron Obstruct Pulmon Dis. 2014;9:597-611. doi:10.2147/COPD.S61854

19. The Japanese Respiratory Society. Guidelines for the diagnosis and treatment of COPD, 4th Edition (in Japanese); 2013. Available from: http://www.jrs.or.jp/. Accessed April 9, 2019.
20. Sasaki H, Nakamura M, Kida K, et al. Reference values for spirogram and blood gas analysis in Japanese adults. J Jpn Respir Soc. 2001;39(5):S1-S17.

21. Ichinose M, Nishimura M, Akimoto M, et al. Tiotropium/olodaterol versus tiotropium in Japanese patients with COPD: results from the DYNAGITO study. Int $J$ Chron Obstruct Pulmon Dis. 2018;13:2147-2156. doi:10.2147/COPD

22. Hashimoto S, Ikeuchi H, Murata S, Kitawaki T, Ikeda K, Banerji D. Efficacy and safety of indacaterol/glycopyrronium in Japanese patients with COPD: a subgroup analysis from the SHINE study. Int J Chron Obstruct Pulmon Dis. 2016;11:2543-2551. doi:10.2147/ COPD

23. Dorinsky P, DePetrillo P, Siddiqui S, Maes A, Reisner C. Safety and pharmacokinetics of budesonide/glycopyrronium/formoterol fumarate dihydrate metered dose inhaler (BGF $\mathrm{MDI}$ ) in healthy adult subjects of Japanese descent. Pulm Pharmacol Ther. 2018;51:18-25. doi:10.1016/j.pupt.2018.05.001

24. Müllerova $\mathrm{H}$, Chigbo $\mathrm{C}$, Hagan $\mathrm{GW}$, et al. The natural history of community-acquired pneumonia in COPD patients: a population database analysis. Respir Med. 2012;106(8):1124-1133. doi:10.1016/j.rmed.2012.04.008

25. Ichinose M, Fukushima Y, Inoue Y, et al. Long-term safety and efficacy of budesonide/glycopyrrolate/formoterol fumarate metered dose inhaler formulated using co-suspension delivery technology in Japanese patients with COPD. Int J Chron Obstruct Pulmon Dis. 2019;14:2993-3002

26. Battisti WP, Wager E, Baltzer L, et al. Good publication practice for communicating company-sponsored medical research: GPP3. Ann Intern Med. 2015;163(6):461-464. doi:10.7326/M15-0288

\section{Publish your work in this journal}

The International Journal of COPD is an international, peer-reviewed journal of therapeutics and pharmacology focusing on concise rapid reporting of clinical studies and reviews in COPD. Special focus is given to the pathophysiological processes underlying the disease, intervention programs, patient focused education, and self management protocols. This journal is indexed on PubMed Central, MedLine and CAS. The manuscript management system is completely online and includes a very quick and fair peer-review system, which is all easy to use. Visit http://www.dovepress.com/testimonials.php to read real quotes from published authors. 